\title{
A Memetic Approach for the Max-Cut Problem
}

\author{
Qinghua Wu and Jin-Kao Hao* \\ LERIA, Université d'Angers, 2 Boulevard Lavoisier, 49045 Angers Cedex 01, France \\ $\{$ wu, hao\}@info.univ-angers.fr
}

\begin{abstract}
The max-cut problem is to partition the vertices of a weighted graph $G=(V, E)$ into two subsets such that the weight sum of the edges crossing the two subsets is maximized. This paper presents a memetic max-cut algorithm (MACUT) that relies on a dedicated multi-parent crossover operator and a perturbation-based tabu search procedure. Experiments on $30 \mathrm{G}$-set benchmark instances show that MACUT competes favorably with 6 state-of-the-art max-cut algorithms, and for 10 instances improves on the best known results ever reported in the literature.
\end{abstract}

Keywords: Multi-parent crossover, memetic algorithm, local search, graph partitioning.

\section{Introduction}

Consider an undirected graph $G=(V, E)$ with vertex set $V=\{1, \ldots, n\}$ and edge set $E \subset V \times V$. Let $w_{i j} \in Z$ be the weight associated with edge $\{i, j\} \in E$. The well-known max-cut problem is to seek a partition of the vertex set $V$ into two disjoint subsets $S_{1} \subset V$ and $S_{2}=V \backslash S_{1}$, such that the weight of the cut, defined as the sum of the weights on the edges connecting the two subsets, is maximized, i.e., $\max \sum_{u \in S_{1}, v \in S_{2}} w_{u v}$. The max-cut problem, more precisely its weighted version, is one of Karp's 21 NP-complete problems [10].

The computational challenge of the max-cut problem has motivated a large number of solution procedures including approximation algorithms, exact methods and metaheuristics. The approximation approach (see for example [5011]) provides a guaranteed performance, but is generally outperformed by other methods in computational testing. Recent examples on exact methods include the cut and price approach [14] and the branch and bound approach [18]. For large instances, various metaheuristic algorithms have been extensively used to find high-quality solutions in an acceptable time. Some representative examples include GRASP [4, ant colony 8], hybrid genetic algorithm [12, tabu search [161321, scatter search [15], global equilibrium search [19] and maximum neural network [20].

In this paper, we present a memetic algorithm for the max-cut problem which is inspired by a very recent algorithm initially designed for the balanced maxbisection problem [22. Experiments on a set of 30 well-known benchmark instances show that our memetic approach performs very well compared with state of the art algorithms.

\footnotetext{
* Corresponding author. 


\section{A Memetic Algorithm for the Max-Cut Problem}

\subsection{Outline of the Memetic Algorithm}

Memetic algorithms are hybrid search methods that typically blend populationbased search and neighborhood-based local search framework. The basic idea behind memetic approaches is to combine advantages of the crossover that discovers unexplored promising regions of the search space, and local optimization that finds good solutions by concentrating the search around these regions. The general architecture of our memetic algorithm for the max-cut problem is summarized in Algorithm 1. From an initial population of solutions which are first improved by a tabu search procedure, the algorithm carries out a number of evolution cycles. At each cycle, which is also called a generation, $m(m \geq 2)$ parents are randomly selected to serve as parents and the crossover operator is applied to create an offspring solution, which is further optimized by tabu search. Subsequently, the population updating rule decides whether the improved offspring should be inserted into the population and which existing individual should be replaced. We describe below the main components of our memetic algorithm.

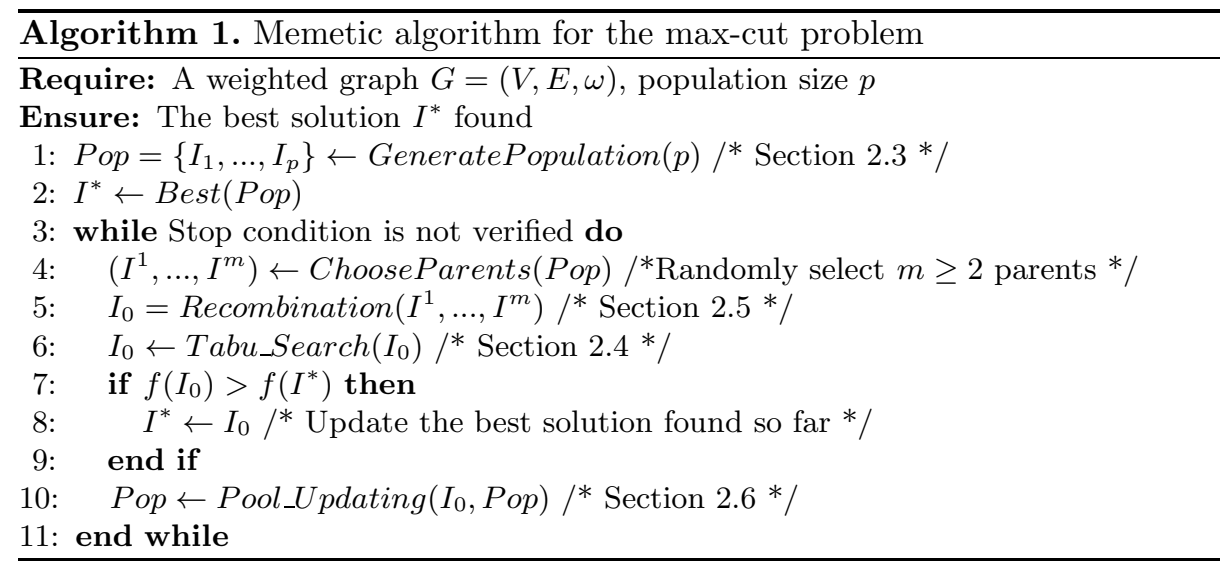

\subsection{Search Space and Fitness Function}

Given a graph $G=(V, E)$ where each edge $\{i, j\} \in E$ is assigned a weight $w_{i j}$, the search space explored by our memetic algorithm is defined as the set of all the partitions of $V$ into 2 disjoint subsets, i.e., $\Omega=\left\{\left\{S_{1}, S_{2}\right\}: S_{1} \cap S_{2}=\right.$ $\left.\emptyset, S_{1} \cup S_{2}=V\right\}$. For a given partition or cut $I=\left\{S_{1}, S_{2}\right\}$, its fitness $f(I)$ is the weight of the cutting edges crossing $S_{1}$ and $S_{2}$, i.e.,

$$
f(I)=\sum_{i \in S_{1}, j \in S_{2}} w_{i j}
$$




\subsection{Initial Population}

The initial population of size $p$ is constructed as follows. For each individual, we first assign randomly the vertices of the graph to the two vertex subsets $S_{1}$ and $S_{2}$ to produce a starting solution, and then apply the tabu search improvement procedure (see section 2.4) to obtain a local optimum. The resulting solution is added to the population if the solution does not duplicate any solution in the population. This procedure is repeated until $2 \times p$ solutions are obtained from which we retain the $p$ best ones to form the initial population.

\subsection{Perturbation-Based Tabu Search Improvement}

To improve the newly generated offspring created by the crossover, we apply a perturbation-based tabu search procedure which integrates a periodic perturbation mechanism to bring diversification into the search. The general procedure of our tabu search method is described in Algorithm 2, Starting from an given solution, the tabu search procedure is first used to optimize the solution as far as possible until the best solution found so far cannot be improved within a certain number of iterations (lines $6-12$ ), then the perturbation mechanism is applied to the current solution to generate a new starting solution (line 13-15), whereupon a new round of tabu search is launched. This process is repeated until a maximum allowed number (MaxIter) of iterations is reached.

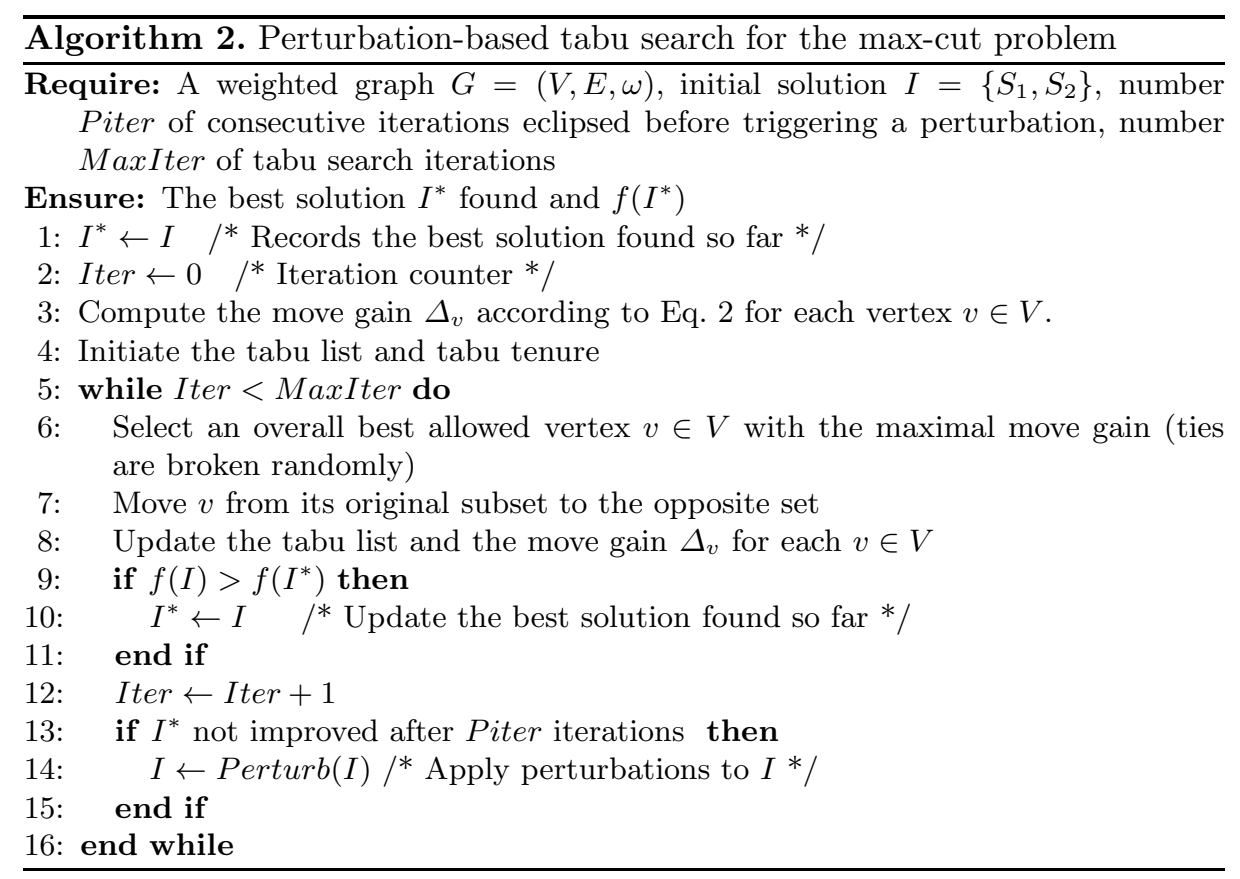


Our tabu search procedure employs a neighborhood defined by the simple oneflip move, which consists of moving a vertex $v \in V$ from its original subset to the opposite set. Notice that this neighborhood is larger than the neighborhood used in 22 where the move operator displaces consecutively two vertices between the two subsets of the current solution to keep the partition balance.
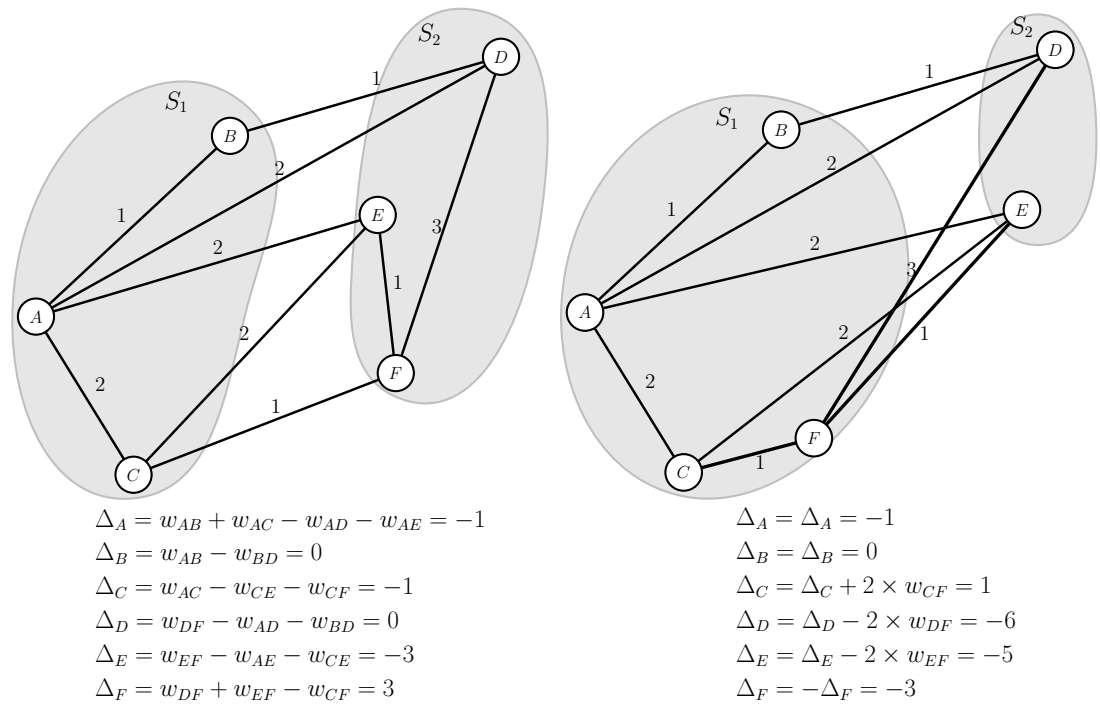

Fig. 1. An example of the initialization (left) and update (right) of the move gain

The concept of move gain is used to represent the change in the fitness function $f$ (Eq. 1, Section 2.2). It expresses how much a cut could be improved if a vertex $v$ is moved from its subset to the other subset. In our implementation, we employ a streamlined incremental technique for fast evaluation of move gains. Specifically, let $\Delta_{v}$ be the move gain of moving vertex $v$ to the other subset. Then initially, each move value can be calculated in linear time using the following formula (see Figure 1 (left)).

$$
\Delta_{v}= \begin{cases}\sum_{x \in S_{1}, x \neq v} w_{v x}-\sum_{y \in S_{2}} w_{v y}, & \text { if } v \in S_{1} \\ \sum_{y \in S_{2}, y \neq v} w_{v y}-\sum_{x \in S_{1}} w_{v x}, & \text { otherwise. }\end{cases}
$$

Each time one displaces a vertex $v$ from its set to the other set, one just needs to update a subset of move gains affected by this move by applying the following abbreviated calculation (see Figure1 (right)):

1. $\Delta_{v}=-\Delta_{v}$

2. for each $u \in V-\{v\}$,

$$
\Delta_{u}= \begin{cases}\Delta_{u}-2 \times w_{u v}, & \text { if } u \text { is in the same set as } v \text { before moving } v \\ \Delta_{u}+2 \times w_{u v}, & \text { otherwise. }\end{cases}
$$


Then each iteration of our tabu search procedure selects a move with the largest $\Delta$ value (breaking ties randomly) whcih is not forbidden by the tabu list. Each time a vertex $v$ is moved from its original subset to the opposite subset, $v$ is forbidden to go back to its original set for a certain number $t t$ of iterations ( $t t$ is called the tabu tenure). The tabu tenure is tuned dynamically according to the mechanism described in [6. Finally, a simple aspiration criterion is applied which allows a move to be performed in spite of being tabu if it leads to a solution better than the current best solution.

When the best solution cannot be further improved by tabu search, a perturbation operator is triggered to vary the local optimum solution from which a new round of tabu search is launched. The perturbation consists in randomly moving $\gamma$ vertices from their original subsets to the opposite subsets where $\gamma$ is a parameter which indicates the strength of the perturbation.

\subsection{The Multi-parent Crossover}

It is commonly admitted that, in order to be efficient, a crossover operator should be adapted to the problem being solved and should integrate useful problemspecific knowledge of the given problem.

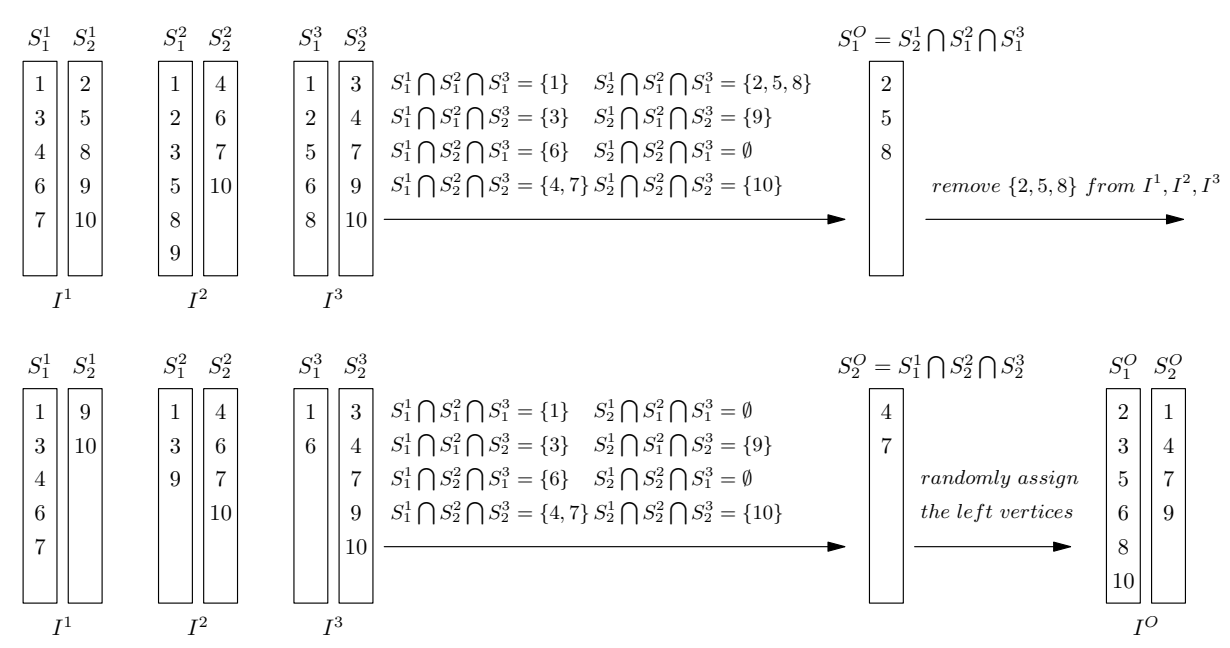

Fig. 2. An example of the multi-parent crossover operator

The max-cut problem is a grouping problem [3], i.e., a cut is composed of two distinct groups of vertices. An important principle in crossover design for grouping or partitioning problems is to manipulate promising groups of objects rather than individual objects. Such an approach for designing crossover operators has been successfully applied to solve a number of grouping problems such as graph coloring [7/17, bin packing [3] and graph partitioning [16]. 
We propose a grouping-based multi-parent crossover for the max-cut problem, the proposed crossover tries to preserve subsets (or grouping vertices) of the vertex partitions which are common to all parent individuals. More formally, given $m$ chosen parents $\left\{I^{1}, \ldots, I^{m}\right\}(m \geq 2$ is chosen randomly from a given range, fixed at $\{2,3,4\}$ in this paper), each cut $I^{i}$ can be represented as $I^{i}=$ $\left\{S_{1}^{i}, S_{2}^{i}\right\}$. Then we produce an offspring solution $I^{O}=\left\{S_{1}^{O}, S_{2}^{O}\right\}$ using these $m$ parent individuals as follows.

We first select one subset from each of the $m$ parents such that the cardinality of intersection of these chosen subsets is maximal. Then we build $S_{1}^{O}$ as the intersection of these $m$ selected subsets, i.e., $S_{1}^{O}=\arg \max \left\{\left|S_{x_{1}}^{1} \cap \ldots \cap S_{x_{m}}^{m}\right|\right.$ : $\left.x_{1}, \ldots, x_{m} \in\{1,2\}\right\}$. When $S_{1}^{O}$ is built, for each $v \in S_{1}^{O}, v$ is removed from all the parent individual subsets in which it occurs. Then, $S_{2}^{O}$ is constructed in the same way as for building $S_{1}^{O}$ such that $S_{2}^{O}=\arg \max \left\{\left|S_{x_{1}}^{1} \cap \ldots \cap S_{x_{m}}^{m}\right|: x_{1}, \ldots, x_{m} \in\right.$ $\{1,2\}\}$. If a vertex $v$ is left unassigned after this procedure, $v$ is placed either to $S_{1}^{O}$ or $S_{2}^{O}$ at random. Figure 2 shows an example with 3 parents.

Notice that this crossover differs from that of [22] for at least two reasons. It operates on multi-parents (instead of 2 parents) and its offspring is not required to be a balanced cut.

\subsection{The Population Updating Rule}

The updating procedure of Pop is invoked each time an offspring solution is created by the crossover operator and then improved by tabu search. Specifically, the improved solution $I^{O}$ is added into Pop if $I^{O}$ is distinct from any solution in Pop and the fitness $f\left(I^{O}\right)$ is higher (better) than the worst solution $I^{w}$ in Pop. Under this circumstance, we update Pop by replacing $I^{w}$ with $I^{O}$.

\section{Computational Results}

\subsection{Experimental Protocol and Benchmark Instances}

Our MACUT algorithm is coded in $\mathrm{C}$ and compiled using GNU GCC on a PC (Pentium 2.83GHz CPU and 8G RAM). We show our results on a selection of 30 well-known G-set benchmark graphs (see Table 1) 14131516 19 21]. The first 24 instances (with at most 3000 variables) are the most popular and we include 6 additional larger instances with 5000 to 10000 variables. The edge weights of these graphs take values in the set $\{-1,0,1\}$.

The parameters of our algorithm are determined by a preliminary experiment on a selection of problem instances and are fixed as follows: population size $p=$ 10, non-improvement tabu search iterations before perturbation Piter $=500$, perturbation strength $\gamma=150$, number of tabu search iterations applied to each offspring MaxIter $=10^{6}$, number of parents for crossover $m \in\{2,3,4\}$. Given the stochastic nature of MACUT, each instance is independently solved 20 times, each run being limited to 30 minutes for graphs with $|V|<5000$ and 120 minutes

\footnotetext{
${ }_{1}$ Available at http://www.stanford.edu/ yyye/yyye/Gset/
} 
for graphs with $|V| \geq 5000$. These timeout limits are comparable with the stop conditions used in $[15521$.

\subsection{Comparisons with the Best Known Results}

Table 1 presents the detailed computational results of our MACUT algorithm as well as its underlying perturbation-based tabu search (PTS). The first two columns in the table indicate the name and the number of vertices of the graph. Column 3 presents the best-known objective value $f_{\text {pre }}$ in the literature [4]13]15]16]19|21]. Columns 4 to 7 show MACUT's results including the best objective value $\left(f_{\text {best }}\right)$, the averaged objective value $\left(f_{\text {avg }}\right)$ over the 20 runs, the success rate (hit) for reaching $f_{\text {best }}$ and the average CPU time in seconds (time) over the 20 runs for which the $f_{\text {best }}$ value is reached. The last 4 columns present the results of its underlying perturbation-based tabu search.

Table 1. Computational results of MACUT and its underlying PTS on 30 G-set maxcut instances

\begin{tabular}{|c|c|c|c|c|c|c|c|c|c|c|}
\hline \multirow[t]{2}{*}{ Instance } & \multirow[t]{2}{*}{$|V|$} & \multirow[t]{2}{*}{$f_{p r e}$} & \multicolumn{4}{|c|}{ MACUT } & \multicolumn{4}{|c|}{$\overline{\mathrm{PTS}}$} \\
\hline & & & $f_{\text {best }}$ & $f_{\text {avg }}$ & hit & time $(s)$ & $f_{\text {best }}$ & $f_{a v g}$ & hit & $\overline{\text { time }(s)}$ \\
\hline$G_{1}$ & 800 & 11624 & 11624 & 11624 & $20 / 20$ & 8.0 & 11624 & 11624 & $20 / 20$ & 6.9 \\
\hline$G_{2}$ & 800 & 11620 & 11620 & 11620 & $20 / 20$ & 6.3 & 11620 & 11620 & $20 / 20$ & 7.5 \\
\hline$G_{3}$ & 800 & 11622 & 11622 & 11622 & $20 / 20$ & 3.0 & 11622 & 11622 & $20 / 20$ & 2.8 \\
\hline$G_{11}$ & 800 & 564 & 564 & 564 & $20 / 20$ & 2.5 & 564 & 564 & $20 / 20$ & 1.9 \\
\hline$G_{12}$ & 800 & 556 & 556 & 556 & $20 / 20$ & 2.5 & 556 & 556 & $20 / 20$ & 4.0 \\
\hline$G_{13}$ & 800 & 582 & 582 & 582 & $20 / 20$ & 3.4 & 582 & 582 & $20 / 20$ & 4.1 \\
\hline$G_{14}$ & 800 & 3064 & 3064 & 3063.95 & $19 / 20$ & 450.0 & 3064 & 3063.9 & $18 / 20$ & 661.2 \\
\hline$G_{15}$ & 800 & 3050 & 3050 & 3050 & $20 / 20$ & 22.1 & 3050 & 3050 & $20 / 20$ & 24.9 \\
\hline$G_{16}$ & 800 & 3052 & 3052 & 3052 & $20 / 20$ & 11.6 & 3052 & 3052 & $20 / 20$ & 10.7 \\
\hline$G_{22}^{10}$ & 2000 & 13359 & 13359 & 13359 & $20 / 20$ & 74.8 & 13359 & 13359 & $20 / 20$ & 206.2 \\
\hline$G_{23}$ & 2000 & 13342 & 13344 & 13344 & $20 / 20$ & 280.0 & 13344 & 13343.2 & $12 / 20$ & 651.7 \\
\hline$G_{24}$ & 2000 & 13337 & 13337 & 13337 & $20 / 20$ & 252.2 & 13337 & 13335.6 & $20 / 20$ & 815.6 \\
\hline$G_{32}$ & 2000 & 1410 & 1410 & 1410 & $20 / 20$ & 349.2 & 1410 & 1408.3 & $3 / 20$ & 844.6 \\
\hline$G_{33}$ & 2000 & 1382 & 1382 & 1382 & $20 / 20$ & 391.4 & 1380 & 1379.6 & $18 / 20$ & 667.6 \\
\hline$G_{34}$ & 2000 & 1384 & 1384 & 1384 & $20 / 20$ & 220.7 & 1384 & 1381.6 & $2 / 20$ & 512.4 \\
\hline$G_{35}$ & 2000 & 7685 & 7686 & 7685.9 & $18 / 20$ & 895.7 & 7676 & 7674.2 & $2 / 20$ & 1400.9 \\
\hline$G_{36}$ & 2000 & 7677 & 7679 & 7676.3 & $6 / 20$ & 1395.4 & 7671 & 7669.3 & $1 / 20$ & 1024.7 \\
\hline$G_{37}$ & 2000 & 7689 & 7690 & 7689.65 & $16 / 20$ & 903.7 & 7678 & 7675.8 & $1 / 20$ & 1175.3 \\
\hline$G_{43}$ & 1000 & 6660 & 6660 & 6660 & $20 / 20$ & 3.6 & 6660 & 6660 & $20 / 20$ & 3.7 \\
\hline$G$ & 1000 & 6650 & 6650 & 6650 & $20 / 20$ & 3.7 & 6650 & 6650 & $20 / 20$ & 3.0 \\
\hline$G_{45}$ & 1000 & 6654 & 6654 & 6654 & $20 / 20$ & 18.2 & 6654 & 6654 & $20 / 20$ & 20.1 \\
\hline$G_{48}$ & 3000 & 6000 & 6000 & 6000 & $20 / 20$ & 0.2 & 6000 & 6000 & $20 / 20$ & 0.2 \\
\hline$G_{49}$ & 3000 & 6000 & 6000 & 6000 & $20 / 20$ & 0.4 & 6000 & 6000 & $20 / 20$ & 0.4 \\
\hline$G_{50}$ & 3000 & 5880 & 5880 & 5880 & $20 / 20$ & 15.0 & 5880 & 5880 & $20 / 20$ & 13.6 \\
\hline$G_{55}$ & 5000 & 10236 & 10299 & 10290.8 & $2 / 20$ & 2496.0 & 10235 & 10221 & $1 / 20$ & 1807.3 \\
\hline$G_{56}$ & 5000 & & 4016 & 4006.9 & $2 / 20$ & 2897.2 & 3954 & 3941.7 & $1 / 20$ & 2108.9 \\
\hline$G_{60}$ & 7000 & 14057 & 14186 & 14171.1 & $1 / 20$ & 5827.1 & 14065 & 14048.4 & $1 / 20$ & 789.3 \\
\hline$G_{65}$ & 8000 & 5518 & 5550 & 5538.7 & $1 / 20$ & 5879.6 & 5488 & 5479.1 & $1 / 20$ & 1476.5 \\
\hline$G_{66}$ & 9000 & 6304 & 6352 & 6331.9 & $1 / 20$ & 6203.8 & 6266 & 6255.2 & $1 / 20$ & 2748.2 \\
\hline$G_{67}^{60}$ & 10000 & 6894 & 6934 & 6922.4 & $1 / 20$ & 6761.3 & 6901 & 6892.1 & $1 / 20$ & 1142.0 \\
\hline
\end{tabular}

From Table1, we observe that MACUT attains the best-known result for each of the 30 graphs. More importantly, MACUT improves on the best known results for 10 instances (indicated in bold). The average computing time required for MACUT to reach its best results $f_{\text {pre }}$ varies from 3 seconds to 1.8 hours. It is clear that the required time to attain the current best-known objective value of column $f_{\text {pre }}$ is shorter for the 10 graphs where MACUT finds improved solutions.

When comparing MACUT with its underlying PTS, one observes that MACUT outperforms PTS in terms of the best and average objective values. Indeed, 
for 10 instances, MACUT is able to achieve better solution with much larger cut values. For 15 instances, MACUT reaches larger average objective values than PTS. In particular, it is remarkable that for each of the instances with at least 5000 vertices, MACUT performs far better than PTS. These comparative results demonstrate that the crossover operator is essential for the success of our MACUT algorithm and help MACUT to discover better solutions that are not attainable by our tabu search algorithm alone.

\subsection{Comparisons with State-of-Art Max-Cut Algorithm}

To further assess the performance of our MACUT approach, we now compare the results of our MACUT algorithm with the most effective heuristic algorithms in the literature. Due to the differences among the programming languages, data structures, compiler options and computers, we do not focus on computing time. Instead, we are mainly interested in solution quality for this experiment. We just mention that the timeout limits we used are quite similar to those adopted by some recent references like [15/21].

Table 2. Comparison with 6 state-of-the-art algorithms in terms of the best results obtained

\begin{tabular}{|c|c|c|c|c|c|c|c|c|}
\hline \multirow[t]{2}{*}{ Instance } & \multirow[t]{2}{*}{$f_{\text {pre }}$} & \multirow[t]{2}{*}{$f_{\text {best }}$} & \multicolumn{6}{|c|}{ Best results of 6 reference max-cut algorithms } \\
\hline & & & GES 19] & SS 15 & TS-UBQP 13 & VNSPR 4 & CirCut[2] & $\begin{array}{c}\text { GRASP- } \\
\text { TS/PM 21 }\end{array}$ \\
\hline$\overline{G_{1}}$ & 11624 & 11624 & 11624 & 11624 & 11624 & 11621 & 11624 & 11624 \\
\hline$G_{2}$ & 11620 & 11620 & 11620 & 11620 & 11620 & 11615 & 11617 & 11620 \\
\hline$G_{3}$ & 11622 & 11622 & 11622 & 11622 & 11620 & 11622 & 11622 & 11620 \\
\hline$G_{11}$ & 564 & 564 & 564 & 562 & 564 & 564 & 560 & 564 \\
\hline$G_{12}$ & 556 & 556 & 556 & 552 & 556 & 556 & 552 & 556 \\
\hline$G_{13}$ & 582 & 582 & 582 & 578 & 580 & 580 & 574 & 582 \\
\hline$G_{14}$ & 3064 & 3064 & 3064 & 3060 & 3061 & 3055 & 3058 & 3063 \\
\hline$G_{15}$ & 3050 & 3050 & 3050 & 3049 & 3050 & 3043 & 3049 & 3050 \\
\hline$G_{16}$ & 3052 & 3052 & 3052 & 3045 & 3052 & 3043 & 3045 & 3052 \\
\hline$G_{22}$ & 13359 & 13359 & 13359 & 13346 & 13359 & 13295 & 13346 & 13349 \\
\hline$G_{23}$ & 13342 & 13344 & 13342 & 13317 & 13342 & 13290 & 13317 & 13332 \\
\hline$G_{24}$ & 13337 & 13337 & 13337 & 13303 & 13337 & 13276 & 13314 & 13324 \\
\hline$G_{32}$ & 1410 & 1410 & 1410 & 1398 & 1406 & 1396 & 1390 & 1406 \\
\hline$G_{33}$ & 1382 & 1382 & 1382 & 1362 & 1378 & 1376 & 1360 & 1374 \\
\hline$G_{34}$ & 1384 & 1384 & 1384 & 1364 & 1378 & 1372 & 1368 & 1376 \\
\hline$G_{35}$ & 7685 & 7686 & 7685 & 7668 & 7678 & 7635 & 7670 & 7661 \\
\hline$G_{36}$ & 7677 & 7679 & 7677 & 7660 & 7660 & 7632 & 7660 & 7660 \\
\hline$G_{37}$ & 7689 & 7690 & 7689 & 7664 & 7664 & 7643 & 7666 & 7670 \\
\hline$G_{43}$ & 6660 & 6660 & 6660 & 6656 & 6660 & 6659 & 6656 & 6660 \\
\hline$G_{44}$ & 6650 & 6650 & 6650 & 6648 & 6639 & 6642 & 6643 & 6649 \\
\hline$G_{45}$ & 6654 & 6654 & 6654 & 6642 & 6652 & 6646 & 6652 & 6654 \\
\hline$G_{48}$ & 6000 & 6000 & 6000 & 6000 & 6000 & 6000 & 6000 & 6000 \\
\hline$G_{49}$ & 6000 & 6000 & 6000 & 6000 & 6000 & 6000 & 6000 & 6000 \\
\hline$G_{50}$ & 5800 & 5800 & 5880 & 5880 & 5880 & 5880 & 5880 & 5880 \\
\hline$G_{55}$ & 10236 & 10299 & - & - & 10236 & - & - & - \\
\hline$G_{56}$ & 3934 & 4016 & - & - & 3934 & - & - & - \\
\hline$G_{60}$ & 14057 & 14186 & - & - & 14057 & - & - & - \\
\hline$G_{65}$ & 5518 & 5550 & - & - & 5518 & - & - & - \\
\hline$G_{66}$ & 6304 & 6352 & - & - & 6304 & - & - & - \\
\hline$G_{67}$ & 6894 & 6934 & - & - & 6894 & - & - & - \\
\hline Better & & & 4 & 18 & 18 & 18 & 19 & 12 \\
\hline Equal & & & 20 & 6 & 12 & 6 & 5 & 12 \\
\hline Worse & & & 0 & 0 & 0 & 0 & 0 & 0 \\
\hline
\end{tabular}


Table 2 compares our MACUT algorithm with 6 state-of-the-art algorithms, which cover the best known results for the tested instances. Columns 2 and 3 recall the previous best known results $\left(f_{\text {pre }}\right)$ and the best results found by MACUT $\left(f_{\text {best }}\right)$. Columns 4 to 9 present the best results obtained by these reference algorithms. The last three rows show the summary of the comparison between our MACUT algorithm and these reference algorithms. The rows 'Better', 'Equal' and 'Worse' respectively denotes the number of instances for which our MACUT algorithm gets better, equal and worse results than the corresponding reference algorithm. From the last three rows of Table 2, it is observed that our MACUT algorithm outperforms the 6 reference algorithms in terms of the quality of the best solution found. In comparison with each of these 6 algorithm, MACUT achieves at least 4 better solutions and in no case, MACUT's result is worse than that of these reference algorithms. This experiment confirms thus the effectiveness of the proposed memetic approach to deliver high quality solutions for the tested 30 benchmark max-cut instances.

\section{Conclusions}

We presented an effective memetic algorithm for the NP-hard max-cut problem. The proposed MACUT algorithm integrates a grouping-based multi-parent crossover which tries to preserve groups of the vertex shared by the parent solutions and a dedicated perturbation-based tabu search procedure. The design of our crossover operator is motivated by an experimental observation (not shown in the paper due to the page limit) that groups of vertices are always shared by high quality solutions. Experimental results confirmed that the crossover operator boosts the performance of the algorithm and helps the search to discover high quality solutions unachievable by a local search algorithm alone. The experiments of MACUT on 30 well-known G-set benchmark instances demonstrated, by providing new best results for 10 instances, its competitiveness compared to 6 state-of-the-art algorithms. Additional studies are needed to better understand the proposed algorithm.

Acknowledgment. We are grateful to the referees for their comments and questions which helped us to improve the paper. The work is partially supported by the RaDaPop (2009-2013) and LigeRO (2010-2013) projects (Pays de la Loire Region, France).

\section{References}

1. Benlic, U., Hao, J.K.: A multilevel memetic approach for improving graph kpartitions. IEEE Transactions on Evolutionary Computation 15(5), 624-642 (2011)

2. Burer, S., Monteiro, R.D.C., Zhang, Y.: Rank-two relaxation heuristics for max-cut and other binary quadratic programs. SIAM Journal on Optimization 12, 503-521 (2001)

3. Falkenauer, E.: Genetic algorithms and grouping problems. Wiley, New York (1998) 
4. Festa, P., Pardalos, P.M., Resende, M.G.C., Ribeiro, C.C.: Randomized heuristics for the max-cut problem. Optimization Methods and Software 7, 1033-1058 (2002)

5. Frieze, A., Jerrum, M.: Improved approximation algorithm for max k-cut and maxbisection. Algorithmica 18, 67-81 (1997)

6. Galinier, P., Boujbel, Z., Fernandes, M.C.: An efficient memetic algorithm for the graph partitioning problem. Annals of Operations Research 191(1), 1-22 (2011)

7. Galinier, P., Hao, J.K.: Hybrid evolutionary algorithms for graph coloring. Journal of Combinatorial Optimization 3(4), 379-397 (1999)

8. Gao, L., Zeng, Y., Dong, A.: An ant colony algorithm for solving Max-cut problem. Progress in Natural Science 18(9), 1173-1178 (2008)

9. Goemans, M.X., Williamson, D.P.: Improved approximation algorithms for maximum cut and satisfiability problems using semidefinite programming. Journal of the Association for Computing Machinery 42(6), 1115-1145 (1995)

10. Karp, R.M.: Reducibility among combinatorial problems. In: Miller, R.E., Thacher, J.W. (eds.) Complexity of Computer Computation, pp. 85-103. Plenum Press (1972)

11. Karish, S., Rendl, F., Clausen, J.: Solving graph bisection problems with semidefinite programming. SIAM Journal on Computing 12, 177-191 (2000)

12. Kim, S.H., Kim, Y.H., Moon, B.Y.: A Hybrid Genetic Algorithm for the MAX CUT Problem. In: Genetic and Evolutionary Computation Conference, pp. 416-423 (2001)

13. Kochenberger, G., Hao, J.K., Lü, Z., Wang, H., Glover, F.: Solving large scale max cut problems via tabu search. Accepted to Journal of Heuristics (2012)

14. Krishnan, K., Mitchell, J.: A semidefinite programming based polyhedral cut and price approach for the Max-Cut problem. Computational Optimization and Applications 33, 51-71 (2006)

15. Marti, R., Duarte, A., Laguna, M.: Advanced scatter search for the max-cut problem. INFORMS Journal on Computing 21(1), 26-38 (2009)

16. Palubeckis, G.: Application of multistart tabu search to the MaxCut problem. Information Technology and Control 2(31), 29-35 (2004)

17. Porumbel, D.C., Hao, J.K., Kuntz, P.: An evolutionary approach with diversity guarantee and well-informed grouping recombination for graph coloring. Computers and Operations Research 37(10), 1822-1832 (2010)

18. Rendl, F., Rinaldi, G., Wiegele, A.: Solving Max-Cut to optimality by intersecting semidefinite and polyhedral relaxations. Mathematical Programming 121, 307-335 (2008)

19. Shylo, V.P., Shylo, O.V.: Solving the maxcut problem by the global equilibrium search. Cybernetics and Systems Analysis 46(5), 744-754 (2010)

20. Wang, J.: An Improved Maximum Neural Network Algorithm for Maximum Cut Problem. Neural Information Processing 10(2), 27-34 (2006)

21. Wang, Y., Lü, Z., Glover, F., Hao, J.K.: Probabilistic GRASP-tabu search algorithms for the UBQP problem. Accepted to Computers and Operations Research (2012), http://dx.doi.org/10.1016/j.cor.2011.12.006

22. Wu, Q., Hao, J.K.: Memetic search for the max-bisection problem. Accepted to Computers and Operations Research (2012) 